\title{
Primary cilium polarity: morphogenetic consequences.
}

\author{
M. Regolini
}

AudioLogic, Department of Bioengineering and Mathematical Modeling. Milan, Italy

Correspondence: Ing.Regolini@gmx.com

\begin{abstract}
In zebrafish inner ear, hair cell orientation in anterior and posterior maculae of the embryonic otic vesicle is different (about $30-40^{\circ}$ ): this is rather unusual in planar polarity mechanism of action, instead suggests that kinocilia may be rotationally polarized. In mice node, the innermost monociliated cells generate a left-ward fluid flow sensed by the immotile primary cilia of Left peri-nodal cells: the Nodal signaling pathway is then expressed asymmetrically, in the Left lateral plate mesoderm, breaking symmetry in visceral organs (situs solitus); however, Right peri-nodal cells also, if artificially excited by a right-ward flow, break symmetry and activate the Nodal cascade, though inverting visceral organ asymmetry (situs inversus); surprisingly, peri-nodal cells prove to be adept at distinguishing flow directionality. Recently, in the Kupffer's vesicle (the zebrafish laterality organ), chiral primary cilia orientation has been described: primary cilia, in the left and right side, are symmetrically oriented, showing a mirror average divergence of about $15-20^{\circ}$ from the midline. This finding, taken together with the mirror behavior of mouse perinodal cells and zebrafish hair cells, champions the idea of primary cilia enantiomerism.
\end{abstract}

\section{Introduction}

The inner ear of birds and mammals is a fascinating model organ for investigating the interplay between Planar Cell Polarity (PCP) and primary cilia (May-Simera et al., 2015; Chacon-Heszele and Chen 2009): vestibular function (balance) is founded on the analysis of bilateral differences in stimuli perception; on the apical surface of hair cells, stereocilia (that are not cilia but actin-based microvilli) form a stereotypical arrangement that determines directional sensitivity: a bundle of stereocilia, ordered by increasing height (the tallest being nearer to the cell margin where a single primary cilium, the kinocilium, is positioned) is arranged like the uppercase letter ' $V$ ' whose orientation, bilaterally symmetric relative to the sagittal plane of the organism in Left and Right ears, rotates (although conserving Left-Right mirror symmetry) in the different segments of the inner ear; this polarized arrangement, that determines the directional sensitivity to acceleration, is a process in which primary cilia (their centrioles/basal bodies and centrosomes) appear to be responsible for the intrinsic polarity of hair cells whereas PCP orchestrates the establishment of global long-range vectorial information: in fact mutant mice for ciliary genes show disordered intracellular morphology of stereocilia bundles but conserve the correct disposal of PCP core complexes; on the contrary, PCP phenotypes have correct arrangements of stereocilia inside each cell (correct ' $\mathrm{V}$ ' configuration) but the orientation of each cell (and its ' $\mathrm{V}$ ' shaped 
stereocilia bundle) is random; likely ciliary genes play a role downstream PCP and are essential for the intrinsic polarity of hair cells (ordered arrangement of stereocilia), while PCP acts upstream controlling cytoskeleton orientation of all hair cells (tissue polarity). As said, hair cell orientation, variable inside the different segments of the inner ear, although bilaterally symmetric in Left and Right ears, is even more meaningful: orientation of ' $V$ ' shaped bundles of stereocilia has been accurately controlled in the zebrafish, organism less complicated than bird or mammals; in zebrafish embryonic inner ear, specialized ciliary cells, 'tether cells', are precursors of hair cells: Tanimoto and colleagues (2011) measured their ' $\mathrm{V}$ ' bundle orientation in anterior and posterior maculae of both Left and Right otic vesicles, finding that it points laterally: measures, taken clockwise from the rostral pole, showed that in Left-sided anterior macula ' $V$ ' bundles are oriented at $263 \pm 22^{\circ}$, in Right at $91 \pm$ $33^{\circ}$; in posterior maculae, ' $V$ ' bundle orientation is different, more posterior-ward: $236 \pm 20^{\circ}$ in Left macula, $138 \pm 22^{\circ}$ in Right); 30-40 degrees of difference between anterior and posterior maculae cannot be an effect of PCP: such value is more compatible with a controlled rotation of kinocilia, posing the question whether primary cilia are circumferentially polarized.

To ascertain and discuss primary cilium polarity and its interplay with PCP, two works, on much less complicated tissues, the embryonic mouse node and zebrafish Kupffer's vesicle, symmetry breaking organs of mice and fish, provide useful information and enlighten about primary cilia structure and monociliated cell histology.

In the mouse node, motile primary cilia of inner cells rotate clockwise (viewed from above their apical side), as in zebrafish Kupffer's vesicle (Okabe et al., 2008), thus producing a fluid flow directed to the Left side of the node: here monociliated peri-nodal 'crown' cells work as mechanosensitive devices (Praetorius and Spring, 2001); Polycystin-2 (PKD2) cation channels, localized on their cilia, are stretch-activated when cilia are bent by the nodal flow (Yoshiba and Hamada 2014) thus activating Nodal pathway expression only in the Left lateral plate mesoderm, and realizing the usual asymmetry of visceral organs (situs solitus). To ascertain the role of the nodal flow, Nonaka and co-workers (2002) and Yoshiba and colleagues (2012) cultured in wide flowchambers several mouse embryos, fixed in parallel rows and correctly oriented so that crown cells of each node were exposed together, and simultaneously subjected, to artificial laminar flows; Right peri-nodal cells, which also have ciliary-positioned PKD2 cation channels (Yoshiba et al., 2012), responded to artificially induced right-ward flows just like Left cells responded to left-ward flows: perinodal cells can distinguish flow directionality, showing mirror differential sensitivity to oppositely directed flows: Left crown cells are excited by left-ward flows, Right cells by right-ward. Moreover, both Left and Right peri-nodal cells, excited by proper flows, trigger asymmetric expression of the Nodal cascade, but inversely: Right crown cells activate the Nodal pathway in the Right lateral plate mesoderm, producing situs inversus, the mirror image of situs solitus: internal organs are mirror shaped and reversed from their usual positions: same genomic pathway, two bilaterally symmetric realizations. It is worth mentioning that these experiments challenge the morphogen hypothesis: node-secreted signaling substances, flow transported, would invest Left and Right cilia of all nodes together and with increasing gradient; the hypothesized chemosensors, anyway, should at least be mirror positioned on Left and Right crown cell cilia.

In the zebrafish Kupffer's vesicle Ferreira and colleagues (2018) measured motile and immotile cilia orientation relative to the midline discovering that, in the left and right side of the early ( 3 somites stage) vesicle, primary cilia orientation is markedly mirror symmetric: $+14^{\circ}$ in the Left side, $-21^{\circ}$ in the Right for motile cilia; immotile cilia of Left and Right hemisphere showed a similar divergent orientation of $\sim 40^{\circ}$, overall more dextral; later (814 somites stage) cilia orientation rotates $\sim 20^{\circ}$ toward the right, showing a dextral orientation over the whole vesicle, yet maintaining the same angular difference between the Left and Right side. Cilia of laterality organs in mice and fish appear, morphologically and physiologically, mirror symmetric. 


\section{Discussion}

In experimental flow chambers, each artificial fluid flow, Left- or Right-ward, bends crown cells cilia of the same node oppositely relative to the embryo (and node) midline: when a left-ward flow bends away from the midline primary cilia of Left peri-nodal cells, these cells respond by opening PKD2 ion channels (then situs solitus), whereas Right crown cells, whose cilia are bent toward the midline, do not respond; the opposite rightward flow bends toward the midline cilia of Left peri-nodal cells, that are not excited, while Right crown cells, whose cilia are bent away from the midline, respond by opening PKD2 ion channels (then situs inversus): perinodal cells respond only to self-ward flows coming from the midline.

The mouse node is an array made up of 150-250 antero-posterior polarized cells (Hashimoto and Hamada, 2010): basal bodies of motile primary cilia must be posterior-positioned for a laminar flow is produced by an ordered array of monociliated cells (Nonaka et al., 2005; Hirokawa et al., 2006): planar cell polarity establishes antero-posterior polarity of nodal cells and provides the posterior positioning and tilt to node cilia (Hashimoto et al. 2010); PCP polarizes antero-posteriorly the cells of Xenopus laevis gastrocoel roof plate, the laterality organ of frogs (Antic et al., 2010) as the dorsal forerunner cells precursors of Kupffer's vesicle of the zebrafish Danio Rerio (Oteiza et al., 2010). As seen, peri-nodal cells, antero-posteriorly polarized and aligned, are excited only when their cilia are bent away from the node midline by self-ward directed flows: the rationale behind their mirror response is that primary cilia of Left and Right crown cells are each other mirror symmetric, so that their PKD2 cation channels, mirror positioned relative to the embryo midline, are excited by oppositely directed flows. This thesis is supported by the cited recent finding of Ferreira and colleagues in the zebrafish Kupffer's vesicle.

Primary cilia are assembled by the mother centriole of an integer centrosome: cells containing only the mother centriole cannot form the primary cilium (Loukil et al., 2017); a role of centrioles and centrosomes in Left-Right patterning is not a new topic: Vanderberg and Levin (2009) suggested that "the coordination of the 3 axes is performed by a cytoskeletal organizing center such as the centriole or basal-body: a sharp midline separation is already evident after the first cell cleavage in Xenopus and Left and Right blastomeres inherit immediately differential chiral information, then transmitted to the progeny" (see also McDowell et al., 2016); Xu and colleagues (2007) proposed an autonomous "intrinsically chiral structure, perhaps the centrosome, serving as a template for managing polarity in the absence of spatial cues: such a template could help to determine LeftRight asymmetry and mirror planar polarity in development". Mouse node and zebrafish Kupffer's vesicle show how centrioles and centrosomes may actually work in Left-Right patterning.

Geimer and Melkonian (2004; 2005) reviewed by Marshall (2012, Pearson, (2014), Dutcher and O'Toole (2016) described inside the basal-body of Chlamydomonas "an 'acorn-like' asymmetric structure, adhering in a highly inter-individual reproducible and invariable manner to triplets N. 2-1-9-8-7" and another structure, shaped like the uppercase letter ' $V$ ' (centrin V-fiber) in contact with triplets N. 9, 5 and 4: "Whereas the cartwheel is thought to nucleate the nine-fold rotational symmetry of the microtubular triplets, the acorn might play an equally important role imposing rotational asymmetry on the microtubular triplets, perhaps leading to the asymmetric assembly of basal-body-associated fibers and hence cellular asymmetry in general" (Geimer and Melkonian, 2004). A marker of "rotational asymmetry" can be arranged clockwise or counter-clockwise like a belt, thus originating two enantiomeric structures, inversely polarized; indeed, it seems that the 9-fold architecture of ciliary locomotive machinery has been utilized to build discrete geometric tools, circumferentially polarized for carrying out finely tuned directional tasks: centriole 9-fold architecture has been adopted by distal and subdistal appendages of mother centrioles, transition fibers of basal bodies, Y-shaped linkers of the cilium transition zone, pericentriolar material of centrosomes, whose 9-scaffold toroidal geometry is sustained by pericentrin (Mennella et al., 2012; Mennella 2014). 
Bilateral symmetry is the main characterizing property of Bilateria: any structure is built as Left- and Righthanded; organ shape depends on the side in which genes of founder cells (primordia or buds) are switched on: sinistral or destral implementation of gene programs is an autonomous process based on the Left or Right cell intrinsic mirror chirality; paws, legs, arms, wings are paired for empowering efficient locomotive systems, and also sensorial organs are paired to guarantee good spatial orientation; unpaired axial organs (skull, chest, vertebral column, urogenital apparatus) are made of two symmetric halves, one Left and one Right; cardiovascular, respiratory and digestive apparatuses are unpaired in that only one apparatus is built because only one is needed, nonetheless are also paired in that they can be built as Left-handed (situs solitus) or Right handed (situs inversus); for better solving difficult problems (circulatory hydrodynamics, or gut length) their organs cannot be axially positioned: they are realized as sinistral and destral (exactly as limbs) by founder cells belonging to both sides, Left and Right, where (Nodal cascade) different genes are switched on in the Left and Right side: both individuals with situs solitus and situ inversus totalis are perfectly healthy; just because only one copy of these apparatuses is needed, it is necessary that only one of the two possible implementations is selected. Bilateral symmetry is imposed in Bilateria embryos immediately and soon 'broken' in some nascent organs: thus, it is not surprising that imposition and 'breakage' of bilateral symmetry is sustained by the same organelles. In amphioxus, manipulating the Nodal cascade, Li and colleagues (2017) obtained '2- Left' or '2Right' phenotypes: '2-Left' mutants duplicate the wild-type left-sided organs and lose the right-sided ones: instead of their typical mouth only on the Left side, '2-Left' mutants have two, mirror symmetric, "paired bilateral mouths". Similarly, in human cardiac isomerism two Right or two Left atria develop, and in both cases, the two atria are mirror-images of each other (Hildreth et al., 2009). in Bilateria, paired or unpaired, internal or external, organs developed from Left-sided funder cells are precise mirror images of the same organs grown from Right-sided primordia: in situs inversus, Right-handed heart and great vessels, respiratory and digestive apparatuses are perfect mirror images of the same Left-handed organs of situs solitus; both the Left and Right sides are capable of forming a half of all cardiac cameras, but, being their identical genes switched on differently (Nodal pathway), Left and Right sided cells accomplish their task differently. On the contrary, axial structures (notochord, neural tube) are formed by forerunner cells, hailing from both the Left and Right side of the embryo and not subjected to Nodal control, and their behavior is perfectly mirror symmetric (fingerprints and human cortical gyri are not considered because non-genetic factors intervene). A question arises: do laterality organs literally 'break' bilateral symmetry, or do they simply establish in which half of the embryo particular genes are allowed (or forbidden) to be expressed, thus determining the Left or Right handedness of organ future shape? Bilateral symmetry of organs in situs solitus/situs inversus supports the second hypothesis; bilateral symmetry is not 'broken': once the side, where founder cells genes are switched on, has been selected, it is consequently established which of the two possible shapes (sinistral or destral) will be achieved. Histological bilateral symmetry of actual tissues and organs (like the zebrafish Kupffer's vesicle and inner ear) together with the mirror functioning of mouse perinodal cells, strongly suggests that chirality of centrioles/centrosomes is central in Left-Right patterning: considering that the same 'ideal, virtual' shape of every organ can be 'actually' implemented as Left-handed (sinistral) or Right-handed (dextral) depending on the side from which each organ derives, mother centrioles appear as chiral tools, bilaterally symmetric, responsible for driving (mirror symmetrically and autonomously) growth and form of organs in Bilateria. One possibility (as crazy as logical, but not counterintuitive) is that, in Left and Right halves of Bilateria, Left ('levo', ' + ') and Right ('dextro', '-') enantiomeric mother centrioles (and then basal bodies, primary cilia, centrosomes) do exist. This may be the reason why organs developed from Left-sided buds are precise mirror images of the same organs grown from Right-sided primordia. After all, centrioles show the highest micro- macro-scale correlation: they organize pericentriolar material, asters and sister asters, and, in mitosis, by the peculiar 
centrosome duplication cycle, the cytoskeleton of daughter cells is patterned upon that of their mother (planar cell chirality).

\section{Conclusion}

Bilateral symmetry and functioning of actual tissues and organs, like the mouse node, zebrafish Kupffer's vesicle and inner ear, supports chirality of centrioles/centrosomes; moreover, an orchestrated interplay of PCP, primary cilia and centrosomes in tissue polarization, much more pronounced than so far thought and capable of reaching highly and finely tuned directional anisotropic (symmetric and antisymmetric) results, emerges. Mice and fish information is a strong challenge to our knowledge. Are stem cells also chiral?

\section{References}

Antic D, Stubbs JL, Suyama K, Kintner C, Scott MP, Axelrod JD. 2010 Planar cell polarity enables posterior localization of nodal cilia and left-right axis determination during mouse and Xenopus embryogenesis. PLoS One. 2;5(2): e8999.

Chacon-Heszele MF, Chen P. 2009 Mouse models for dissecting vertebrate planar cell polarity signaling in the inner ear. Brain research. 1277:130-140.

Dutcher SK, O'Toole ET, 2016 The basal bodies of Chlamydomonas reinhardtii. Cilia. 1;5: 18.

Ferreira RR, Vilfan A, Pakula G, Supatto W, Vermot J.2018 Chiral cilia orientation in the left-right organizer. bioRxiv preprint first posted online Jan. 23, 2018; doi: http://dx.doi.org/10.1101/252502

Geimer S, Melkonian M. 2004 The ultrastructure of the Chlamydomonas reinhardtii basal apparatus: identification of an early marker of radial asymmetry inherent in the basal body. J. Cell Sci. 117, 2663-2674.

Geimer S, Melkonian M. 2005 Centrin scaffold in Chlamydomonas reinhardtii revealed by immunoelectron microscopy. Eukaryot. Cell; 4:1253-63

Hashimoto M, Shinohara K, Wang J, Ikeuchi S, Yoshiba S, Meno C, Nonaka S, Takada S, Hatta H, Wynshaw-Boris A, Hamada H. 2010 Planar polarization of node cells determines the rotational axis of node cilia. Nature Cell Biology 12, 170-176.

Hashimoto M, Hamada H. 2010 Translation of anterior-posterior polarity into left-right polarity in the mouse embryo. Curr Opin Genet Dev. 20:433-7.

Hildreth V, Webb S, Chaudhry B. 2009 Left cardiac isomerism in the Sonic hedgehog null mouse. J. Anat. 214: 894-904.

Hirokawa N, Tanaka Y, Okada Y, Takeda S. 2006 Nodal Flow and the Generation of Left-Right Asymmetry. Cell 125, Pages 33-45.

Li G, Liu X, Xing C, Zhang H, Shimeld SM, Wanga Y. 2017 Cerberus-Nodal-Lefty-Pitx signalling cascade controls left-right asymmetry in amphioxus. Proc. Natl. Acad. Sci. U S A. 114: 3684-3689.

Loukil A, Tormanen K, Sütterlin C. 2017 The daughter centriole controls ciliogenesis by regulating Neurl-4 localization at the centrosome. J Cell Biol. 201608119.

May-Simera HL, Petralia RS, Montcouquiol M. 2015 Ciliary proteins Bbs8 and Ift20 promote planar cell polarity in the cochlea. Development;142:555-566.. 
Marshall, W.F. (2012). Centriole asymmetry determines algal cell geometry. Curr Opin Plant Biol 15, 632-637.

McDowell G, Rajadurai S, Levin M. 2016 From cytoskeletal dynamics to organ asymmetry: a nonlinear, regulative pathway underlies left-right patterning. Phil. Trans. Royal Soc. B.

Mennella V, Keszthelyi B, McDonald K L, Chhun B, Kan F, Rogers GC, Huang B, Agard D A. 2012. Subdiffractionresolution fluorescence microscopy reveals a domain of the centrosome critical for pericentriolar material organization. Nat. Cell Biol. 14, 1159-1168.

Mennella V. 2014 Amorphous no more: subdiffraction view of the Pericentriolar Material architecture, Trends Cell Biol, 24, 188-197.

Nonaka S, Shiratori H, Saijoh Y, Hamada H. 2002 Determination of left-right patterning of the mouse embryo by artificial nodal flow.

Nonaka S, Yoshiba S, Watanabe D, Ikeuchi S, Goto T, Marshall WF, Hamada H. 2005 De novo formation of leftright asymmetry by posterior tilt of nodal cilia. PLoS Biol. 3, e268. 10.1371.

Okabe N, Xu B, Burdine RD. 2008 Fluid dynamics in zebrafish Kupffer's vesicle. Dev Dyn ; 237(12): 3602-3612.

Oteiza P, Köppen M, Krieg M, Pulgar E, Farias C, Melo C, Preibisch S, Müller D, Tada M, Hartel S, Heisenberg CP, Concha ML. 2010 Planar cell polarity signalling regulates cell adhesion properties in progenitors of the zebrafish laterality organ. Development, 137:3459-68.

Pearson, C.G. 2014 Choosing sides--asymmetric centriole and basal body assembly. J Cell Sci 127, 2803-2810.

Praetorius H.A., Spring K.R. 2001 Bending the MDCK primary cilium increases intracellular calcium. J. Membrane Biol. 184:71-79.

Tanimoto M, Ota Y, Inoue M, Oda Y.2011 Origin of Inner Ear Hair Cells: Morphological and Functional Differentiation from Ciliary Cells into Hair Cells in Zebrafish Inner Ear. Journal of Neuroscience, 31 3784-3794.

Vandenberg LN, Levin M. 2009 Perspectives and open problems in the early phases of left-right patterning. Semin. Cell Dev. Biol. 20: 456-463.

Xu J, Van Keymeulen A, Wakida NM, Carlto P, Berns MW, Bourne HR. 2007 Polarity reveals intrinsic cell chirality. Proc. Natl. Acad. Sci. USA 104: 9296-9300.

Yoshiba S, Hamada H. 2014 Roles of cilia, fluid flow, and Ca2+ signalling in breaking of left-right symmetry.

Yoshiba S, Shiratori H, Y. Kuo IY, Aiko Kawasumi A, Shinohara K, Nonaka S, Asai Y, Sasaki G, Belo JA, Sasaki H, Nakai J, Dworniczak B, Ehrlich BE, Pennekamp P, Hamada H. 2012 Cilia at the node of mouse embryos sense fluid flow for left-right determination via Pkd2. Science 338(6104): 226-231. 